\title{
Impacto en un programa de trasplante renal de un protocolo de donación tras muerte cardiaca controlada con soporte de ECMO
}

\author{
José Luis Cobo Sánchez ${ }^{1}$ M $^{a}$ Jesús Mantilla Gordovil2, Elvira Inmaculada Benito Concha ${ }^{3}$, Esther Liaño \\ Fernández ${ }^{3}$, Eduardo Miñambres García ${ }^{4}$, Raquel Pelayo Alonso ${ }^{5}$ \\ ${ }^{1}$ Máster en Ciencias de la Enfermería. Área de Calidad. Formación. I+D+i de Enfermería. Hospital Universitario \\ Marqués de Valdecilla. Santander. España. ${ }^{2}$ Máster Universitario en Dirección y Gestión Servicios Sanitarios y \\ Sociales. Unidad de Cuidados Intensivos Generales. Hospital Universitario Marqués de Valdecilla. Santander. \\ España. ${ }^{3}$ Diplomado en Enfermería. Unidad de Cuidados Intensivos Generales. Hospital Universitario Marqués \\ de Valdecilla. Santander. España. ${ }^{4}$ Doctor en Medicina. Unidad de Cuidados Intensivos Generales. Hospital \\ Universitario Marqués de Valdecilla. Santander. España. ${ }^{5}$ Diplomado en Enfermería. Servicio de Nefrología. \\ Hospital Universitario Marqués de Valdecilla. Santander. España
}

\section{Resumen}

Objetivo: Analizar el impacto de un protocolo de donación tras muerte cardiaca controlada con soporte de sistema de oxigenación de membrana extracorpórea (PMCC-ECMO) en el programa de trasplante renal de nuestro centro.

Material y Método: Estudio retrospectivo, observacional, descriptivo en una cohorte de injertos renales (IR) procedentes de un PMCC-ECMO. Se evalúan los IR procedentes de 8 pacientes con enfermedad irreversible (EI) en los que se aplica limitación del esfuerzo terapéutico y se consideran como potenciales donantes durante el último semestre del 2014 y el primer trimestre de 2015. Se excluyeron los IR enviados fuera de la comunidad. Se evaluaron indicadores del proceso de donación (IPD) y resultados clínicos de los injertos (RCI).

Resultados: IPD: $100 \%$ hombres, edad media 60 años, tiempo medio agónico 9,37 minutos; causa EI 62,5\% de origen pulmonar, $37,5 \%$ de origen neurológico. Se obtuvieron 13 riñones, 3 hígados y 2 pulmones: 2 IR fuera de la comunidad. Los IR provenientes del PMCC-ECMO supusieron un $20 \%$ de los IR del 2014 en nuestro centro. RCI: De los 11 IR trasplantados en nuestro centro, sólo 1 no fue viable por trombosis de

Correspondencia:

José Luis Cobo Sánchez

Área de Calidad, Formación, Investigación y Desarrollo Hospital Universitario Marqués de Valdecilla

Avda. Valdecilla, s/n. 39008 Santander

E-mail:jocobo@humv.es las venas renales, y otro IR se retrasó la función renal por causas inmunológicas. Valores medios función IR al mes: creatinina sérica $1,88 \mathrm{mg} / \mathrm{dl}$, aclaramiento creatinina $56,82 \mathrm{~mL} / \mathrm{min}$, urea $0,798 \mathrm{~g} / \mathrm{L}$.

Conclusiónes: EI PMCC-ECMO ha tenido un alto impacto en el programa de trasplante renal, tanto por incrementar la tasa de donación, como por la alta tasa de viabilidad de los injertos.

PALABRAS CLAVE: obtención de tejidos y órganos (métodos); atención de enfermería; trasplante renal; donación de órganos tras la muerte cardiaca; donantes tipo III de Maastricht.

Impact of a donor protocol on a renal transplant program after controlled cardiac death with ECMO support

\section{Abstract}

Aim: To analyze the impact of a donor protocol after controlled cardiac death with support of extracorporeal membrane oxygenation (PMCC-ECMO) in the renal transplant program of our center.

Material and method: Retrospective, observational, descriptive study in a cohort of renal grafts (RG) from PMCC-ECMO. RGs from 8 patients with irreversible disease (IE) in which therapeutic effort limitation is applied are evaluated and considered as potential 
donors during the last six months of 2014 and the first quarter of 2015. IRs sent out of the community were excluded. Indicators of the donation process (IDP) and clinical results of the grafts (CRG) were evaluated.

Results: IDP: $100 \%$ men, mean age 60 years, mean agonizing time 9.37 minutes; cause of IE: $62.5 \%$ of pulmonary origin, $37.5 \%$ of neurological origin. We obtained 13 kidneys, 3 livers and 2 lungs: 2 RG outside the community. RGs from the PMCC-ECMO accounted for $20 \%$ of the total in 2014 at our center. CRG: Of the 11 RGs transplanted in our center, only 1 was not viable due to thrombosis of the renal veins, and another $R G$ presented delayed renal function due to immunological causes. Mean values of RG function per month: serum creatinine $1.88 \mathrm{mg} / \mathrm{dl}$, creatinine clearance $56.82 \mathrm{~mL}$ / min, urea $0.788 \mathrm{~g} / \mathrm{L}$.

Conclusions: PMCC-ECMO has had a high impact on the renal transplant program, both for increasing the donation rate and for the high viability rate of the grafts.

KEYWORDS: tissue and organ procurement (methods); nursing care; kidney transplantation; organ donations after cardiac death; type III Maastricht donors.

\section{Introducción}

En España, según datos de la Organización Nacional de Trasplantes, en el año 2014 la tasa de donación se situó en 30,8 donantes efectivos p.m.p., con cifras similares desde 2010, en donde hubo una disminución significativa ${ }^{1}$. Cantabria se sitúa a la cabeza en cuanta a tasa de donaciones a nivel nacional en los dos últimos años, pero ésta ha descendido (55, 8 p.m.p. en 2013 a 52,7 p.m.p. en 2014)1,2. Esta situación está íntimamente ligada a la disminución de la mortalidad por siniestralidad de tráfico y por enfermedad cardiovascular, como consecuencia de una mejor seguridad vial y atención al paciente neurocrítico.

Respecto al número de trasplantes renales (TR), éste se ha estancado en torno a los 2500 trasplantes al año en España desde el 2011. En 2013 a nivel estatal, el $23,6 \%$ de los riñones generados no pudieron trasplantarse ${ }^{3}$. En Cantabria el número de TR se duplicó entre el año 2012 y el 2013, por un aumento en el número de donantes vivos y por la implantación de un progra- ma de donación en asistolia, con buenos resultados en cuanto a tiempos y viabilidad de los injertos ${ }^{3,4}$.

A pesar de esta buena situación en nuestra comunidad, la demanda de la lista de espera del programa de trasplante renal aumenta a un ritmo mayor ${ }^{5}$. Esta situación da lugar al planteamiento de estrategias dirigidas a aumentar la accesibilidad al trasplante, que fundamentalmente son 4: optimización de la donación en muerte encefálica, potenciar el programa de donante vivo, considerar el donante con criterios expandidos y desarrollar programas de donación en asistolia (ver tabla 1$)^{6}$. En este sentido, en nuestro hospital se ha puesto en marcha un protocolo de donación tras muerte cardiaca (donante tipo III de Maastricht), con la peculiaridad del uso de un sistema de oxigenación de membrana extracorpórea (ECMO).

Nuestro objetivo es analizar el impacto de un programa de donación tras muerte cardiaca controlada con soporte de ECMO (PMCC-ECMO) en el programa de TR de nuestro centro.

Tabla 1. Clasificación de los donantes a corazón parado según clasificación de Mastricht modificada - Madrid $2011^{6}$

Donación asistolia no controlada

- I Fallecidos fuera del hospital: Incluye víctimas de una muerte súbita, traumática o no, acontecidas fuera del hospital que, por razones obvias, no son resucitadas.

- II Resucitación infructuosa: Incluye pacientes que sufren una parada cardiaca y son sometidos a maniobras de reanimación que resultan no exitosas. En esta categoría se diferencian dos subcategorías:

o II.a. Extrahospitalaria: La parada cardiaca ocurre en el ámbito extrahospitalario y es atendida por el servicio de emergencias extrahospitalario, quien traslada al paciente al hospital con maniobras de cardio-compresión y soporte ventilatorio.

o II.b. Intrahospitalaria: La parada cardiaca ocurre en el ámbito intrahospitalario, siendo presenciada por el personal sanitario, con inicio inmediato de maniobras de reanimación.

\section{Donación asistolia controlada}

- III A la espera del paro cardiaco: Incluye pacientes a los que se aplica limitación del tratamiento de soporte vital tras el acuerdo entre el equipo sanitario y éste con los familiares o representantes del enfermo.

- IV Paro cardiaco en muerte encefálica: Incluye pacientes que sufren una parada cardiaca mientras se establece el diagnóstico de muerte encefálica o después de haber establecido dicho diagnóstico, pero antes de que sean llevados a quirófano. Es probable que primero se trate de restablecer la actividad cardiaca pero, cuando no se consigue, puede modificarse el proceso al de donación en asistolia. 


\section{Material y Método}

Estudio retrospectivo, observacional, descriptivo en una cohorte de injertos renales (IR) procedentes de un PMCC-ECMO de nuestro centro entre Junio de 2014 y Marzo de 2015. Se evalúan los IR procedentes de 8 pacientes con enfermedad irreversible (EI) en los que se aplica limitación del esfuerzo terapéutico y se consideran como potenciales donantes.

EI PMCC-ECMO incluye a aquellos pacientes ingresados en la Unidad de Cuidados Intensivos (UCI) con enfermedad grave irreversible y sin perspectiva de evolución a muerte encefálica. La secuencia de actuación es la siguiente: El equipo de UCI junto con la familia decide la limitación terapéutica del soporte vital (LTSV). Llegado este momento de decisión, es cuando el equipo de coordinación de trasplantes plantea la posibilidad de donación a la familia. De esta forma la posibilidad de conflicto ético o de intereses desaparece pues se ha separado la limitación del esfuerzo terapéutico de la petición de órganos.

Una vez aceptada la donación, la familia es informada de todo el proceso y se le pedirá el consentimiento informado para la canulación de la ECMO y la administración de fármacos antemortem. Interviene entonces el equipo de cirugía cardiovascular para la canulación la ECMO (catéter fogarty introducido en la arteria femoral con balón en aorta torácica descendente, para excluir de la circulación los órganos torácicos).

Es el momento ahora de ofrecer a la familia la posibilidad del acompañamiento para su despedida. Una situación delicada en la que juega un papel muy importante la enfermera de UCI junto al equipo de coordinación de trasplantes porque es el momento en el que se procede a la retirada del soporte vital.

A partir de la extubación es fundamental el control de la monitorización de las constantes vitales y del tiempo en el que la tensión arterial sistólica esté por debajo de $60 \mathrm{mmHg}$, un tiempo denominado Tiempo Agónico o tiempo de isquemia caliente funcional que debe ser inferior a 60 minutos para asegurar la viabilidad de los órganos (se aconseja $<30-60$ minutos para hígado $\mathrm{y}<$ 120 minutos para riñón $)^{7}$.

Tras 5 min de parada cardiorrespiratoria el medico intensivista certifica la muerte y es ahora cuando se inicia la preservación de los órganos, conectando la ECMO para normotermia $\left(37^{\circ} \mathrm{C}\right)$ y la despedida de la familia para el traslado a quirófano y extracción por laparatomía de los órganos abdominales.

Se excluyeron del análisis los IR enviados fuera de la comunidad. Se evaluaron indicadores del proceso de donación (IPD) y resultados clínicos de los injertos (RCI). Al ser un reducido número de casos se muestran datos descriptivos y no se realiza inferencia estadística.

\section{Resultados}

En el periodo de estudio se realizaron 11 TR con injertos procedentes de 8 donantes del programa de PMCC-ECMO.

Las características clínicas y los IPD de cada uno de los donantes se presentan en la tabla 2: 100\% hombres,

Tabla 2. Características de los donantes del protocolo de donación tras muerte cardiaca controlada con soporte de ECMO.

\begin{tabular}{|c|c|c|c|c|c|c|c|c|}
\hline & Donante 1 & Donante 2 & Donante 3 & Donante 4 & Donante 5 & Donante 6 & Donante 7 & Donante 8 \\
\hline Edad & 65 & 53 & 70 & 51 & 69 & 64 & 46 & 67 \\
\hline Causa exitus & ACVA-T & $\mathrm{AOP}$ & $\mathrm{AOP}$ & ACVA-T & $\mathrm{AOP}$ & APPCR & ACVA-T & $\mathrm{AOP}$ \\
\hline Días en UCI & 5 & 8 & 5 & 5 & 8 & 6 & 6 & 13 \\
\hline $\begin{array}{l}\text { Tiempo LTSV-PCR } \\
\text { (minutos) }\end{array}$ & 10 & 9 & 11 & 8 & 8 & 16 & 6 & 20 \\
\hline $\begin{array}{l}\text { Tiempo Agónico-PCR } \\
\text { (minutos) }\end{array}$ & 7 & 6 & 10 & 5 & 7 & 14 & 4 & 17 \\
\hline Tiempo ECMO (minutos) & 104 & 151 & 204 & 145 & 129 & 103 & - & 125 \\
\hline Órganos donados & RR & RR & RI & $\mathrm{RR}, \mathrm{H}$ & $\mathrm{RR}, \mathrm{H}$ & RR & PP & $\mathrm{RR}, \mathrm{H}$ \\
\hline
\end{tabular}

ACVA-T: accidente cerebrovascular del tronco. APPCR: anoxia post parada cardiorrespiratoria. UCI: unidad de cuidados intensivos. LTSV: limitación de las técnicas de soporte vital. PCR: parada cardiorrespiratoria. ECMO: sistema de oxigenación de membrana extracorpórea. A0P: alarma cero de trasplante pulmonar. RR: riñones. RI: riñón izquierdo. H: Hígado. PP: pulmones. 
edad media 60 años, tiempo medio agónico 9,37 minutos; causa EI $62,5 \%$ de origen pulmonar, $37,5 \%$ de origen neurológico, media de estancia en UCI 7 días (rango entre 5 y 13 días). El tiempo medio entre la LTSV y el inicio del tiempo agónico (tiempo que transcurre desde la retirada de las medidas hasta que la TAS baja por debajo de $60 \mathrm{~mm} \mathrm{Hg}$ y se produce la assistolia) fue de 11 minutos (rango entre 6 y 20 minutos). El tiempo medio entre el tiempo agónico y la parada cardiorrespiratoria (tiempo que transcurre desde que la TAS baja por debajo de $60 \mathrm{~mm} \mathrm{Hg} \mathrm{y} \mathrm{la} \mathrm{asistolia)} \mathrm{fue} \mathrm{de} 8,75$ minutos (rango entre 4 y 17 minutos). En todos los casos, el tiempo de isquemia caliente estuvo muy por debajo de las 2 horas (máximo 25 minutos, mínimo 11 minutos).

Se obtuvieron 13 riñones, 3 hígados y 2 pulmones: 2 IR fuera de la comunidad. Los IR provenientes del PMCC-ECMO supusieron un $20 \%$ de los IR del 2014 en nuestro centro.

De los 11 IR trasplantados en nuestro centro, sólo 1 no fue viable por trombosis de las venas renales, y otro IR se retrasó la función renal por causas inmunológicas. Los valores medios analíticos de los IR al mes fueron: creatinina sérica media $1.88 \mathrm{mg} / \mathrm{dl}$ (rango $1-2,7 \mathrm{mg} / \mathrm{dl}$ ), aclaramiento creatinina medio $56,82 \mathrm{~mL} / \mathrm{min}$ (rango $130-22,3 \mathrm{~mL} / \mathrm{min}$ ), Urea 0,798 $\mathrm{g} / \mathrm{L}$ (rango 0,3-1,39 g/L). Las características clínicas y evolución analítica de los receptores se muestran en la tabla 3. Uno de los pacientes presentó rechazo humoral, precisando tratamiento con plasmaférisis e inmunoglobulinas endovenosas, presentando una creatinina sérica al $2^{\circ}$ mes de $2,3 \mathrm{mg} / \mathrm{dl}$ y al $3^{\text {er }}$ mes de $1,9 \mathrm{mg} / \mathrm{dl}$.

\section{Discusión}

EI PMCC-ECMO en nuestro centro ha supuesto un incremento en el número de IR disponibles en nuestra comunidad, sin detrimento del programa de donación en muerte encefálica (ME). En algunos países como Reino Unido o los Países Bajos, la puesta en marcha de un programa de donantes tipo III de Maastrich ha tenido un efecto de vasos comunicantes: los donantes en asistolia tipo III aumentaban mientras que los de ME disminuían ${ }^{8,9}$. Un adecuado seguimiento e instauración de un programa de donación en asistolia controlado sin detrimento de la donación por ME, por parte de la coordinación de trasplantes, ha de permitir maximizar el pool total de órganos.

En nuestro país se han descrito series de IR procedentes de programas de donantes tipo III de Maastrich. Portolés y cols presentaron 10 casos con una mediana de creatinina sérica al mes de $2,1 \mathrm{mg} / \mathrm{dl}$ (rango 0,7-3,2 $\mathrm{mg} / \mathrm{dl}$ ) con un caso de rechazo agudo ${ }^{10}$. Frutos y cols publicaron 6 casos de trasplante renal, con peores valores analíticos al mes (creatinina sérica media $3,34 \mathrm{mg} / \mathrm{dl}$, rango 1,3 a $5,5 \mathrm{mg} / \mathrm{dl}$ ) y a los 6 meses (creatinina sérica media $1,96 \mathrm{mg} /$ $\mathrm{dl}$, rango 0,9 a 2,7 $\mathrm{mg} / \mathrm{dl}$ ) comparados con nuestra

Tabla 3. Características de los pacientes trasplantados de riñón en nuestro centro y de su evolución analítica.

\begin{tabular}{|c|c|c|c|c|c|c|c|c|c|c|}
\hline & 1 & 2 & 3 & 4 & 5 & 6 & 7 & 8 & 9 & 10 \\
\hline Edad & 63 & 43 & 64 & 63 & 27 & 46 & 63 & 56 & 56 & 63 \\
\hline $\mathrm{N}^{\circ}$ de Trasplante & $1^{\circ}$ & $2^{\circ}$ & $2^{\circ}$ & $1^{\circ}$ & $1^{\circ}$ & $1^{\circ}$ & $2^{\circ}$ & $1^{\circ}$ & $1^{\circ}$ & $2^{\circ}$ \\
\hline Nefropatía & GN & GN & EVR & EVR & PNF & ND & ERV & EP & EP & GN \\
\hline Meses en TRS & 20 & 0 & 6 & 35 & 26 & 13 & 11 & 11 & 4 & 9 \\
\hline $\begin{array}{l}\text { Creatinina sérica } \\
\text { al } 7^{\circ} \text { día }(\mathrm{mg} / \mathrm{dl})\end{array}$ & 5,5 & 1,2 & 1,5 & 1,2 & 3,8 & 3,77 & 3,1 & 4,5 & 4,9 & 2,9 \\
\hline $\begin{array}{l}\text { Creatinina sérica } \\
\text { al } 14^{\circ} \text { día }(\mathrm{mg} / \mathrm{dl})\end{array}$ & 3,2 & 1,3 & 1,2 & 1,3 & 1,9 & 2,9 & 3,7 & 2,7 & 2,8 & 3,1 \\
\hline $\begin{array}{l}\text { Creatinina sérica } \\
\text { al } 1^{\text {er }} \text { mes }(\mathrm{mg} / \mathrm{dl})\end{array}$ & 1,3 & 1 & 1,2 & 1,1 & 1,2 & 1,9 & $3,1^{*}$ & 2 & 2 & 2,7 \\
\hline $\begin{array}{l}\text { Urea sérica } \\
\text { al } 1^{\text {er }} \text { mes }(g / L)\end{array}$ & 0,45 & 0,55 & 0,86 & 0,61 & 0,3 & 0,8 & 1,26 & 0,85 & 0,91 & 1,39 \\
\hline $\begin{array}{l}\text { Aclaramiento creatinina } \\
\mathrm{I}^{\text {er }} \mathrm{mes}(\mathrm{mL} / \mathrm{min})\end{array}$ & 74,04 & 97,2 & 25 & 113,6 & 94,44 & 35,45 & 22,3 & 35,56 & 40,1 & 30,55 \\
\hline
\end{tabular}

GN: glomerulonefritis. EVR: enfermedad vascular renal. PNF: pielonefritis. ND: Nefropatía diabética. EP: Enfermedad poliquística.

*Rechazo humoral que precisó plasmaférisis + inmunoglobulinas e.v. Creatinina sérica al $2^{\circ} \mathrm{mes} 2,3 \mathrm{mg} / \mathrm{dl}$, al $3^{\text {er }} \mathrm{mes} 1,9 \mathrm{mg} / \mathrm{dl}$. 
serie $^{11}$. Estas diferencias se pueden dar por varios factores, pero principalmente por 2 relacionados en el proceso de donación: tiempos de isquemia caliente funcional mayores y el no uso de la ECMO para revertir el tiempo isquémico (posibilidad que ya apuntaba Frutos en su artículo).

La ECMO en sus distintas modalidades, presenta múltiples aplicaciones clínicas, entre ellas, como soporte circulatorio para la perfusión y preservación de órganos para el trasplante en donantes en asistolia' ${ }^{12,13}$. A nivel mundial 3 grupos han publicado sus resultados en un programa de trasplante renal con órganos procedentes de un programa de donación en asistolia utilizando ECMO en distintas modalidades ${ }^{14-18}$. EI grupo del Hospital Nacional de Taipei publicó un análisis retrospectivo de 31 IR procedentes de donantes en asistolia (Maastrich II, III y IV), perfundidos en hipotermia, y los compararon con 219 donantes en ME y 68 donantes vivos, no observando diferencias significativas respecto a la supervivencia de los IR a los 5 años entre ambas modalidades. Este análisis, sin embargo, no distingue entre los 3 tipos de donantes de la categorización de Maastrich ${ }^{14,15}$. El grupo de Michigan presentó un estudio retrospectivo de 24 IR perfundidos en normotermia y sus resultados se compararon con 100 IR de donantes en $M E$, con un catéter que ocluía la aorta y preservaba la circulación abdominal, como en nuestra serie. El retraso en la función renal fue del $24 \%$ en el grupo de ME y del $8,3 \%$ en grupo con ECM016,17. El tercer grupo, de Carolina del Norte, publicaron un estudio retrospectivo comparando 19 IR de un programa de donación en asistolia con ECMO perfundido a $22^{\circ} \mathrm{C}$ y 53 sin el uso de ECMO: encontrando mejor funcional al primer mes en el grupo que usaba la ECMO comparado con el que no (aclaramiento de creatinina $50 \pm 25 \mathrm{ml} / \mathrm{min}$ vs $38 \pm 18 \mathrm{ml} / \mathrm{min} ; \mathrm{p}<0,002)^{18}$.

Estos estudios, junto con la comparación de nuestros resultados con otros programas de trasplante renal con donantes en asistolia controlada sin el uso de ECMO a nivel nacional, parecen indicar la mejor evolución de los mismos utilizando ECMO como soporte circulatorio. En nuestro caso, su uso no sólo ha permitido mejorar los tiempos relacionados con el proceso de donación, sino que además permite a las familias tener la posibilidad de acompañar al paciente en un entorno más propicio, con personal de UCI que ya conocen; y no en un espacio hostil para las familias como puede ser un quirófano.

\section{Conclusiones}

EI PMCC-ECMO ha tenido un alto impacto en el programa de trasplante renal, tanto por incrementar la tasa de donación, como por la alta tasa de viabilidad de los injertos.

$$
\begin{aligned}
& \text { Recibido: } 26 \text { julio } 2017 \\
& \text { Revisado: } 31 \text { julio } 2017 \\
& \text { Modificado: } 4 \text { agosto } 2017 \\
& \text { Aceptado: } 9 \text { agosto } 2017
\end{aligned}
$$

\section{Bibliografía}

1. Organizacion Nacional de Trasplantes. Actividad de donación España 2014.. Madrid; 2014. [Consultado 4 Junio 2016]. Disponible en: http://www.ont.es/ infesp/Memorias/Memoria donantes 2014.pdf

2. Organización Nacional de Trasplantes. Memoria Donación en España 2013. Madrid; 2013. [Consultado 4 Junio 2016]. Disponible en: http:// www.ont.es/infesp/Memorias/Memoria donacion 2013.pdf

3. Organización Nacional de Trasplantes. Actividad de trasplante renal en España 2013. Madrid; 2013. [Consultado 4 Junio 2016] Disponible en: http:// www.ont.es/infesp/Memorias/Memoria trasplante renal 2013.pdf

4. Miñambres E, Suberviola B, Guerra C, Lavid N, Lassalle $\mathrm{M}$, González-Castro $\mathrm{A}$, et al. Experiencia de un programa de donación en asistolia Maastricht II en una ciudad de pequeño tamaño: resultados preliminares. Med Intensiva. 2015 0ct;39(7):433-41.

5. Registro Español de Enfermos Renales. Informe de Diálisis y Trasplante 2013. Madrid; 2013. [Consultado 4 Junio 2016]. Disponible en: http:// www.senefro.org/modules/webstructure/files/ inforreercongsen2014final.pdf

6. Matesanz Acedos R, Coll Torresl E, Domínguez-Gi González B, Perojo Vega L. DONACIÓN EN ASISTOLIA EN ESPAÑA: SITUACIÓN ACTUAL Y RECOMENDACIONES (Documento de Consenso Nacional 2012). Madrid; 2012. [Consultado 4 Junio 2016]. Disponible en: http://www.ont.es/infesp/ DocumentosDeConsenso/DONACIÓN EN ASIST0LIA EN ESPAÑA. SITUACIÓN ACTUAL Y RECOMENDACIONES.pdf 
7. Escudero D, Otero J. Medicina intensiva y donación de órganos. ¿Explorando las últimas fronteras? Med Intensiva. 2015;39(6):366-74.

8. Kompanje EJO, de Groot YJ, Bakker J. Is organ donation from brain dead donors reaching an inescapable and desirable nadir? Transplantation. 15 de julio de 2011;91(11):1177-80.

9. Summers DM, Counter C, Johnson RJ, Murphy PG, Neuberger JM, Bradley JA. Is the increase in DCD organ donors in the United Kingdom contributing to a decline in DBD donors? Transplantation. Diciembre 2010;90(12):1506-10.

10. Portolés-Pérez J, Rubio-Muñoz JJ, LafuenteCovarrubias 0, Sánchez-Sobrino B, CarballidoRodríguez J, Rodríguez-Reina G, et al. Development of a program for kidney transplants using organs donated from donors awaiting cardiac arrest (type III Maastricht). Nefrologia. 2012;32(6):754-9.

11. Frutos-Sanz MÁ, Guerrero-Gómez F, Daga-Ruiz D, Cabello-Díaz M, Lebrón-Gallardo M, QuesadaGarcía G, et al. Kidney transplantation with grafts from type III Maastricht non-beating-heart donors. Nefrologia. 2012;32(6):760-6.

12. Noble DW, Bloomfield R. Problem Solving in Intensive Care -The Role of Extracorporeal Technologies. J Intensive Care Soc. Enero 2014;15(1):4-7.

13. Barrou B, Billault C, Nicolas-Robin A. The use of extracorporeal membranous oxygenation in donors after cardiac death. Curr Opin Organ Transplant. 2013;18(2):148-53.
14. Lee C-Y, Tsai M-K, Ko W-J, Chang C-J, Hu $\mathrm{R}-\mathrm{H}$, Chueh $\mathrm{S}-\mathrm{C}$, et al. Expanding the donor pool: use of renal transplants from non-heartbeating donors supported with extracorporeal membrane oxygenation. Clin Transplant. Julio 2005; 19(3):383-90.

15. Ko WJ, Chen YS, Tsai PR, Lee PH. Extracorporeal membrane oxygenation support of donor abdominal organs in non-heart-beating donors. Clin Transplant. Mayo 2000;14(2):152-6.

16. Gravel MT, Arenas JD, Chenault R, Magee JC, Rudich S, Maraschio M, et al. Kidney transplantation from organ donors following cardiopulmonary death using extracorporeal membrane oxygenation support. Ann Transplant. Enero 2004;9(1):57-8.

17. Magliocca JF, Magee JC, Rowe SA, Gravel MT, Chenault $\mathrm{RH}$, Merion RM, et al. Extracorporeal support for organ donation after cardiac death effectively expands the donor pool. J Trauma. Julio 2005;58(6):1095-102.

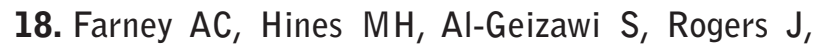
Stratta RJ. Lessons learned from a single center's experience with 134 donation after cardiac death donor kidney transplants. J Am Coll Surg. Mayo 2011;212(4):440-51. 